\title{
Calcification of intervertebral discs in the dachshund: a radiographic and histopathologic study of 20 dogs Øyvind Stigen*1 and Øyvor Kolbjørnsen ${ }^{2}$
}

\author{
Address: ${ }^{1}$ Department of Companion Animal Clinical Sciences, Norwegian School of Veterinary Science, PO Box 8146, 0033 Oslo, Norway and \\ ${ }^{2}$ Department of Pathology, National Veterinary Institute, PO Box 8156, 0033 Oslo, Norway \\ Email: Øyvind Stigen* - oyvind.stigen@veths.no; Øyvor Kolbjørnsen - oyvor.kolbjornsen@vetinst.no \\ * Corresponding author
}

Published: 21 December 2007

Acta Veterinaria Scandinavica 2007, 49:39 doi:10.1/86/1751-0147-49-39
Received: 26 April 2007

Accepted: 21 December 2007

This article is available from: http://www.actavetscand.com/content/49/1/39

(c) 2007 Stigen and Kolbjørnsen; licensee BioMed Central Ltd.

This is an Open Access article distributed under the terms of the Creative Commons Attribution License (http://creativecommons.org/licenses/by/2.0), which permits unrestricted use, distribution, and reproduction in any medium, provided the original work is properly cited.

\begin{abstract}
Background: The purpose of the study was to compare radiographic and histopathologic findings with regard to number and extent of calcified discs in the dachshund.

Methods: The intervertebral discs of 20 dachshunds were subjected to a radiographic and histopathologic examination. The dogs were selected randomly from clinical cases euthanased for reasons unrelated to research at the Norwegian School of Veterinary Science. Lateral radiographs were taken of the vertebral columns after removing them from the carcasses. The histopathologic examination included $5 \mu \mathrm{m}$ thick sections in the transverse plane, stained with hematoxylin-eosin and von Kossa. Radiographs and histological sections were evaluated independently.

Results: A total of 148 (28.5\%) calcified discs were identified at the radiographic and 230 (45.7\%) at the histopathologic examination. Of 92 discs found to be calcified by histopathology, but not by radiography, the degree of calcification was evaluated as 'slight' in 84 (91.3\%). All the intervertebral discs $(n=138)$ that were found to be calcified by radiography were also found to be calcified by histopathology.
\end{abstract}

Conclusion: A sensitivity of 0.6 and specificity of 1.0 for radiography was calculated when using histopathology as the gold standard.

\section{Background}

Intervertebral disc disease (IDD) is the most common cause of neurologic dysfunction in the dog [1]. The disease is most often diagnosed in dachshunds, and in 8117 canine cases of IDD, Priester [2] found $3898(48.0 \%)$ to occur in this breed. The breed prevalence of IDD in the dachshund has been estimated to be $19.0 \%$ [3].

In dachshunds and other chondrodystrophoid breeds, IDD is mainly due to extrusion of nuclear material from the disc into the vertebral canal. Hansen [4] classified this type of disc disease as protrusion of type 1 and found chondroid degenerative changes of the disc with subsequent dystrophic calcification of the nucleus to be significant preceding factors. In accordance with this, radiographic studies have shown that young dachshunds with calcified intervertebral discs are predisposed to IDD $[5,6]$.

The occurrence of calcified discs is found to differ between dachshunds of the same age, size and coat variety [7]. Furthermore, a genetic factor is shown to be essential for the 
occurrence of calcified discs in a dog and the heritability in Norwegian dachshunds is estimated to be 0.15 and 0.22 [8]. In Danish wirehaired dachshunds Jensen and Christensen [9] estimated the heritability to be 0.60 and 0.87 . Thus, the potential exists for a breeding programme that could reduce the occurrence of calcified discs and thereby IDD in this breed.

Low-field magnetic resonance (MR) imaging is found to be very sensitive in the evaluation of disc degeneration in dogs [10]. However, the cost of MR imaging is high and the number of MR machines for veterinary usage is limited. For screening purposes, a radiographic examination is therefore still the most suitable method for the identification of calcified discs in a large number of live dachshunds. Information obtained by reading radiographs can be included as part of a breeding programme.

Different degrees of calcification are found in degenerated chondrodystrophoid discs [11,12]. Discs with minor calcifications could be difficult to identify on radiographs. Also, non-calcified discs could be misjudged and read as calcified. By this, false-positive and false-negative errors may be made in reading radiographs for calcified discs. To the authors' knowledge the extent of such errors has not been reported. However, in a pathologic study of 16 oneyear old chondrodystrophoid dogs Hansen [4] found calcified discs in at least $10(62.5 \%)$ of the dogs, while in a radiographic study of 327 one-year old dachshunds Stigen [7] found calcified discs in 79 (24.2\%) of the dogs. On the assumption that most of the dogs in the former study were dachshunds, these two studies together indicate that a radiographic study is far less sensitive than a pathologic study in identification of calcified discs. The present study was performed to test this hypothesis.

\section{Methods}

The vertebral columns were obtained from 20 dachshunds that were euthanased for reasons unrelated to research at the Department of Companion Animal Clinical Sciences, Norwegian School of Veterinary Science. The case material, including size and coat varieties, sex, age and reason for euthanasia is presented in Table 1 . The age of the dachshunds ranged from ten months to 13 years (mean 5.3 years) and there were twelve (60.0\%) females.

The vertebral columns were separated from the skull, ribs and pelvis by dearticulation. The tail, including the coccygeal vertebrae, was also removed. The spines were freed of muscle, loose connective tissue and ligaments.

Within 24 hours of death, conventional lateral radiographs were taken of the cervical, thoracic, lumbar and sacral vertebral columns [13]. The radiographic equipment was a Philips Medio 50 CP generator and a Super Rotalix 2550 tube. The cassettes were Cawo $18 \times 24 \mathrm{~cm}$ rectangle $\mathrm{x}$-ray cassettes containing a green-emitting 100 MR (fine) intensifying screen on one side of a Kodak TMat L/RA-film. No grid was used and at exposure the specimens were in direct contact with the cassettes. The voltage

Table I: Case material.

\begin{tabular}{|c|c|c|c|c|}
\hline \multirow[t]{2}{*}{ Dog no. } & Variety & \multirow[t]{2}{*}{ Sex } & \multirow[t]{2}{*}{ Age, years } & \multirow[t]{2}{*}{ Reason for euthanasia } \\
\hline & Size, Coat & & & \\
\hline I & Dwarf, Smoothcoated & ㅇ & 8 & Neurological disorder \\
\hline 2 & Dwarf, Longhaired & $\stackrel{+}{+}$ & $<1$ (10 months) & Vascular ring anomaly \\
\hline 3 & Dwarf, Longhaired & $\mathrm{O}^{+}$ & 5 & Intoxication \\
\hline 4 & Dwarf, Longhaired & q & 6 & Neurological disorder \\
\hline 5 & Dwarf, Longhaired & $0^{2}$ & 6 & Multiple fractures \\
\hline 6 & Dwarf, Longhaired & q & 7 & Multiple fractures \\
\hline 7 & Dwarf, Longhaired & O & 13 & Pelvic fracture \\
\hline 8 & Standard, Smoothcoated & $\mathrm{O}^{7}$ & $<1$ (10 months) & Neurological disorder \\
\hline 9 & Standard, Smoothcoated & q & 5 & Neurological disorder \\
\hline 10 & Standard, Smoothcoated & $\mathrm{d}^{7}$ & 6 & Neurological disorder \\
\hline 11 & Standard, Smoothcoated & $0^{7}$ & 8 & Complications to perineal hernia \\
\hline 12 & Standard, Wirehaired & $\gamma^{7}$ & $<$ I (II months) & Owner's request \\
\hline 13 & Standard, Wirehaired & q & 2 & Owner's request \\
\hline 14 & Standard, Wirehaired & $\stackrel{+}{q}$ & 4 & Neurological disorder \\
\hline 15 & Standard, Wirehaired & $\stackrel{+}{q}$ & 4 & Neurological disorder \\
\hline 16 & Standard, Wirehaired & q & 5 & Pulmonary neoplasm \\
\hline 17 & Standard, Wirehaired & o & 7 & Neurological disorder \\
\hline 18 & Standard, Wirehaired & $0^{7}$ & 10 & Owner's request \\
\hline 19 & Standard, Longhaired & $\sigma^{\pi}$ & 3 & Multiple fractures \\
\hline 20 & Standard, Longhaired & q & 4 & Neurological disorder \\
\hline
\end{tabular}


and milliampere-seconds used depended upon the size of the vertebrae and varied between 50 and $60 \mathrm{kV}$ and 10 and $20 \mathrm{mAs}$ respectively.

At least four exposures were taken of each dog covering the vertebral column from the first cervical (C1) to the first sacral (S1) vertebra. The total number of calcified discs and their location in the vertebral column were recorded according to earlier described methods $[13,14]$. Three degrees of calcification of the individual discs were noted and defined as follows (Figure 1):

\section{SLIGHT:}

- one or more calcified bodies with diameter less than 2 $\mathrm{mm}$, or

- calcification of part of the transitional zone between the nucleus pulposus and the annulus fibrosus, or

- indistinct calcification of a larger part of the nucleus pulposus

\section{MODERATE:}

- calcified bodies with diameter less than $2 \mathrm{~mm}$ and calcification of part of the transitional zone between the nucleus pulposus and the annulus fibrosus, or

- distinct calcification of a larger part of the nucleus pulposus, or

- indistinct calcification of the entire nucleus pulposus

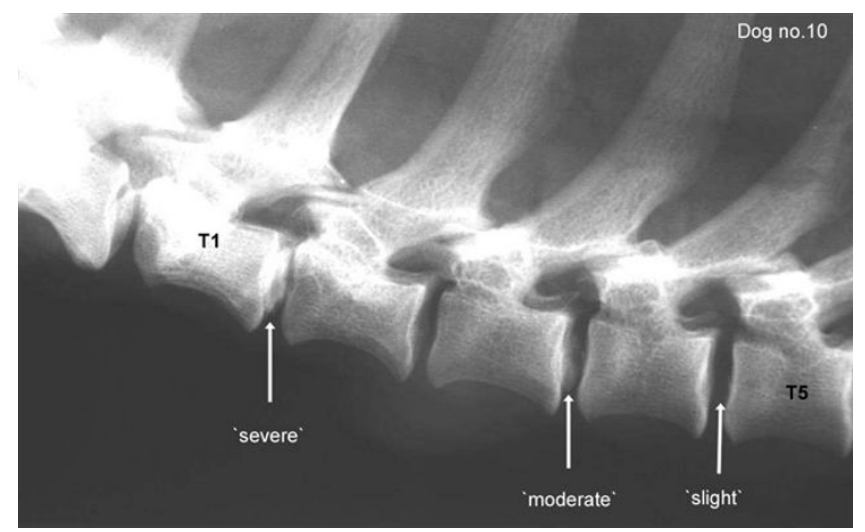

Figure I

Lateral radiograph from the seventh cervical (C7) to the fifth thoracic (T5) vertebra in a six-year-old, male, smoothcoated dachshund of standard size (Dog no. 10). Three intervertebral discs; TI-2, T3-4 and T4-5, shows a 'severe', 'moderate' or 'slight' degree of calcification (arrows).

\section{SEVERE:}

- distinct calcification of the entire nucleus pulposus

If there was doubt about calcification or the degree of calcification, additional radiographs were taken of the vertebral section of current interest.

Immediately after the radiographic examination, specimens for histological examination were collected by removing each intervertebral disc between the second cervical (C2) and first sacral (S1) vertebra in all of the 20 vertebral columns. With tissue forceps, a scalpel handle and no. 11 blade the discs were carefully separated from the adjacent vertebral body end plates (extremitas cranialis et caudalis) and removed.

All specimens (520 discs) were fixed in 4\% phosphatebuffered formaldehyde, $\mathrm{pH}$ 7.2. Subsequently the discs were dehydrated in ethanol, equilibrated in xylene and embedded in paraffin. Decalcifications of the specimens were not performed. The discs were sectioned transversely at about $5 \mu \mathrm{m}$ and stained with hematoxilin-eosin and von Kossa, a method for demonstration of calcium [15]. During the preparation process all sections concerning 17 discs became incomplete and consequently these discs were regarded as withdrawals. By this, 503 discs were available for a final histopathologic examination by light microscopy.

Three degrees of calcification of the individual discs were noted, and for each category, all findings might appear either separately or in combination with other lesions. The degrees of calcification were defined as follows:

\section{SLIGHT (Figure 2):}

- single or multiple foci of calcification occupying less than half of the area of the nucleus pulposus

- single or multiple small foci of calcification within the annulus fibrosus

MODERATE (Figure 3):

- single or multiple foci of calcification occupying more than half, but not all, of the area of the nucleus pulposus

- single or multiple medium size foci of calcification within the annulus fibrosus

- thin calcified ring, continuous or discontinuous, either surrounding the nucleus pulposus or localised within the annulus fibrosus 


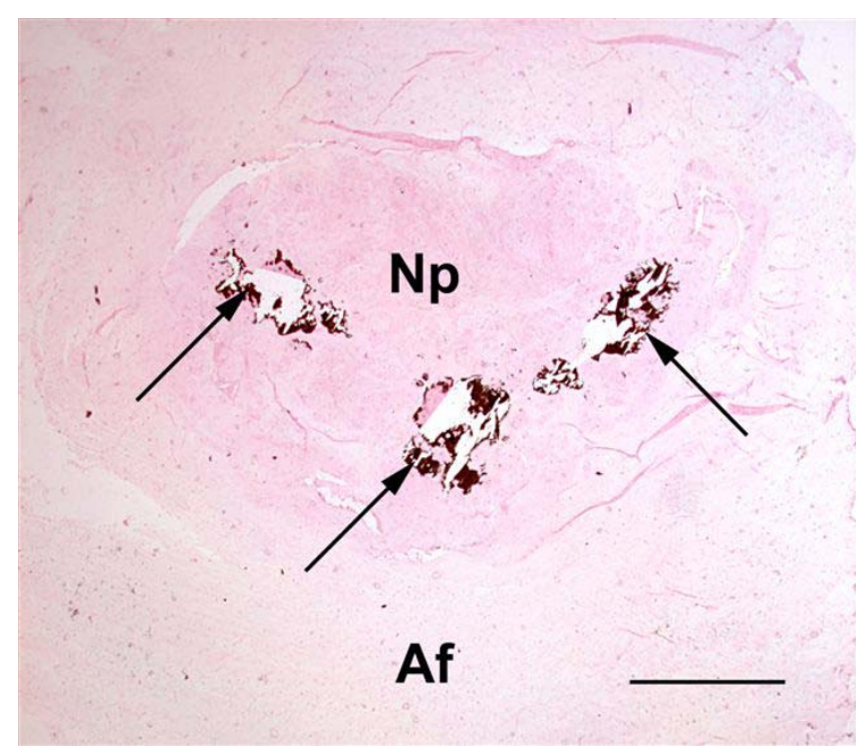

Figure 2

Histopathology of disc no. 6 (C7-TI) from Dog no. 10. Calcium deposits (arrows) are seen multifocally occupying less than half of the area of the nucleus pulposus (Np): 'slight' degree of calcification (I). Af = annulus fibrosus. Von Kossa; Bar $=1.6 \mathrm{~mm}$.

\section{SEVERE (Figure 4):}

- widespread or total calcification of the nucleus pulposus

- single or multiple large foci of calcification within the annulus fibrosus

- broad calcified ring, continuous or discontinuous, either surrounding the nucleus pulposus or localised within the annulus fibrosus

If two or more sections of the same disc were read to have different degrees of calcification, the section with the most severe degree of calcification determined the evaluation of that disc.

Radiographs and histological sections were evaluated independently.

\section{Results}

At the radiographic examination, calcification was found in $148(28.5 \%)$ of the 520 discs. Of all affected discs, a severe degree of calcification was found in 15 , a moderate degree in 43 and a slight degree in 90 discs. Calcified discs were identified in $18(90.0 \%)$ of the dogs and the number of calcified discs in each affected dog ranged from one (dog no. 19) to 20 (dog no. 15).

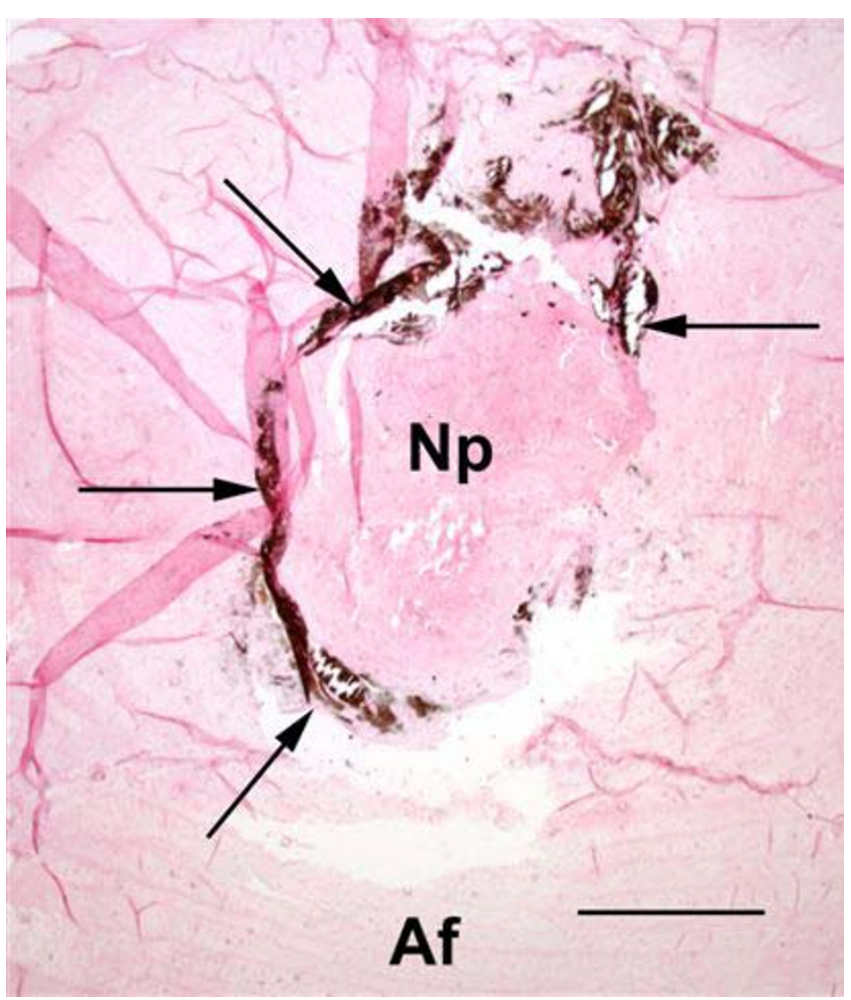

Figure 3

Histopathology of disc no 10 (T4-5) from Dog no. 10. Calcium deposits (arrows) form a thin discontinuous ring surrounding the nucleus pulposus $(\mathrm{Np})$, with one focus extending into the annulus fibrosus: 'moderate' degree of calcification (2). Af = annulus fibrosus. Von Kossa; Bar = 1.6 $\mathrm{mm}$.

At the histopathologic examination, calcification was found in $230(45.7 \%)$ of the 503 discs. Of all affected discs, a severe degree of calcification was found in 30 , a moderate degree in 45 and a slight degree in 155 discs. Calcified discs were identified in all the dogs and the number of calcified discs in each dog ranged from one (dog no. 19) to 24 (dog no. 17).

The individual number and location of calcified discs together with the degree of calcification of each affected disc, identified both by radiography and histopathology, are presented in Additional file 1, Table 3.

Comparative results by radiographic and histopathologic examination concerning 503 discs are presented in Table 2. Of all the discs found to be calcified by histopathology $(\mathrm{n}=230), 138(60.0 \%)$ were found to be calcified by radiography. All the discs found to be calcified by radiography ( $\mathrm{n}=138$ ) were also found to be calcified by histopathology. Using histopathology as the gold standard, a sensitiv- 


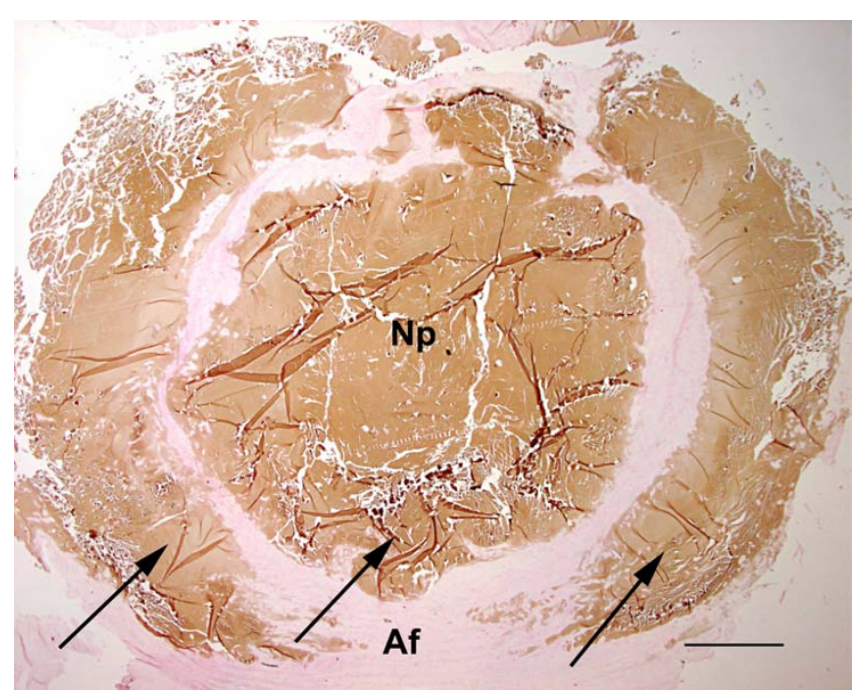

Figure 4

Histopathology of disc no. 7 (TI-2) from Dog no. 10. Calcium deposits (arrows) in the nucleus pulposus $(\mathrm{NP})$ and in a broad discontinuous ring within the annulus fibrosus (Af): 'severe' degree of calcification (3). Von Kossa; Bar = $1.6 \mathrm{~mm}$.

ity of $0.6(138 / 230)$ and a specificity of $1.0(273 / 273)$ was calculated for the radiographic examination.

In 95 discs the degree of calcification was found to be equal in histopathology and radiography. In 125 discs the degree of calcification was higher in histopathology and in 10 it was higher in radiography.

\section{Discussion}

The present study shows that a significantly higher number (45.7\% versus $27.4 \%$ ) of calcified discs are identified by a histopathologic examination compared to a radiographic examination of intervertebral discs in dachshunds. This finding verifies the hypothesis presented at the beginning of the present paper.

Table 2: The distribution of 503 intervertebral discs on the basis of calcification (+) or not (-) evaluated by a radiographic and a histopathologic examination.

\begin{tabular}{|c|c|c|c|c|}
\hline & & \multicolumn{2}{|c|}{ Histolopathology } & \multirow[t]{2}{*}{ Total } \\
\hline & & + & - & \\
\hline \multirow[t]{2}{*}{ Radiology } & + & I 38 (27.4\%) & 0 & I 38 (27.4\%) \\
\hline & - & $92(18.3 \%)$ & $273(54.3 \%)$ & 365 (72.6\%) \\
\hline Total & & $230(45.7 \%)$ & $273(54.3 \%)$ & 503 (100.0\%) \\
\hline
\end{tabular}

At the radiographic examination, a total of 148 calcified discs were identified in 18 dogs. This makes a mean of 8.2 calcified discs in each affected individual. Both the percentage number of dogs with calcified discs $(90.0 \%)$ and the mean number of such discs in each affected dog are higher than values for corresponding parameters found in previous radiographic studies $[6,7,12]$. The fact that the present study is related to clinical cases, whereas the previous radiographic studies were related to clinically normal dachshunds, could be the reason for this disparity.

In dogs no. 2 and 3, no calcified discs were found at the radiographic examination, but at the histopathologic examination a slight degree of calcification was found in five and 15 discs, respectively. This finding shows that a total absence of calcified discs visible on radiographs of a dachshund is no guarantee for the dog not having extensive calcification at histopathologic examination.

Of the 92 discs that were found to be calcified only at the histopathologic examination, $84(91.3 \%)$ were noted to have a 'slight' degree, eight a 'moderate' degree and none a 'severe' degree of calcification. This agrees with the general understanding that an ordinary radiographic examination of the vertebral column in dogs is not very sensitive at detecting minor calcifications of intervertebral discs.

The radiographic examination in the present study was done on vertebral columns separated from the skull, ribs and pelvis and freed of soft tissue. This meant that superimposition of tissue adjacent to the discs (e.g. caput costae and os ilium) was avoided on the radiographs. Also motion blurring was not a problem with the examination of dead specimens. These factors contributed to high technical quality of the radiographs. Therefore, the sensitivity of the radiographic examination in the present study is likely higher than could be expected in similar studies on live dogs. As a consequence, the presented results concerning total number of calcified discs and degree of calcification of individual discs, cannot directly be compared to results from previous radiographic studies $[5-7,9,12]$ on live dogs.

The 20 dachshunds included in the present study were euthanased up to 24 hours before being available for the radiographic examination. As a result, rigor mortis was present in several dogs and for these the standard procedure for spinal radiography of live dogs $[13,16]$ was inappropriate. Two dogs (no. 3 and 18) had been subjected to post-mortem examinations immediately after euthanasia and their vertebral columns were thereafter included in the study. To achieve radiographs of high technical quality from more dogs, separated vertebral columns, instead of vertebral columns "in situ", were chosen for radiography. Diagnostic imaging of separated vertebral columns is pre- 
viously reported in radiographic $[17,18]$ and MR [10] examinations in dogs.

Radiographs and histological sections were each evaluated by one person, which means that both the radiographic and the histopathologic examination were subject to significant interobserver variation. With two or more persons evaluating the radiographs and the histological sections respectively, the interobserver variation and consequently the standard deviations of the observations presented in Table 2, would decrease. Consequently, the calculated sensitivity of 0.6 represents an underestimate.

At the radiographic examination, additional radiographs were taken if there was any doubt about calcification. At the histopathologic examination, at least two complete histological sections of each disc were examined to remove any doubt about calcification. By this, the present study should not be encumbered with significant intraobserver variation.

A specificity of 1.0 indicates that no false-positive errors were made at the radiographic examination. The high technical quality of the radiographs is probably a significant reason for this. Another reason could be a reserved attitude by the reader of the radiographs, evaluating discs with uncertain calcification as negative.

Von Kossa, a special stain for identification of calcium, was included in the histopathologic examination of all intervertebral discs. In previous histopathologic examinations of disc degeneration in dogs $[4,10]$, this stain was not used. In the study by Seiler et al. [10], decalcification was also part of the preparation for histopathologic examination. A discrepancy in results can easily arise when different studies adopt different protocols.

In the present study, histopathology was found considerably more sensitive than radiography in identifying calcified discs. Nevertheless, in ten discs the degree of calcification was found to be higher in radiography than in histopathology. A histopathologic examination of non representative sections could be an explanation for the different findings in these ten discs.

\section{Conclusion}

A histopathologic examination is superior to a radiographic examination in identification of calcified intervertebral discs in the dachshund. A sensitivity of 0.6 and a specificity of 1.0 for radiography is calculated using number of calcified discs as parameter and histopathology as the gold standard. Calcification of the nucleus pulposus and/or the annulus fibrosus is a significant sign of disc degeneration in dachshunds and the distribution of detectable calcification in affected discs represents the distribution of a continuous variable.

\section{Competing interests}

The author(s) declare that they have no competing interests.

\section{Authors' contributions}

$\varnothing S$ conceived of the study, carried out the radiographic examination and is the main author of the paper. ØK carried out the histopathologic examination. Both authors read and approved the final manuscript.

\section{Additional material}

\section{Additional file 1}

Table 3. Degree of calcification, evaluated by a radiographic and a histopathologic examination in 26 intervertebral discs from each of 20 dachshunds. Degrees of calcification evaluated by radiography: light grey: slight. dark grey: moderate. black: severe. Degrees of calcification evaluated by histopathology: 1 slight. 2 moderate. 3 severe. Other note: -: intervertebral disc not available for a final histopathologic examination. Click here for file

[http://www.biomedcentral.com/content/supplementary/17510147-49-39-S1.doc]

\section{Acknowledgements}

We are grateful to Ms. Birgit Røe and Ms. Anne-Kristine Fevang for performing the histopathologic sections, to Dr. Agnar Kvellestad for practical help with photographic editing and to Dr. Monica Theresa Johnsen for language consultation.

\section{References}

I. Seim HB: Thoracolumbar disk disease: diagnosis, treatment and prognosis. Canine Pract 1995, 20(I):8-13.

2. Priester WA: Canine intervertebral disc disease - occurrence by age, breed, and sex among 8.1 I 7 cases. Theriogenology 1976, 6:293-303.

3. Ball MU, McGuire JA, Swaim SF, Hoerlein BF: Patterns of occurrence of disk disease among registered dachshunds. J Am Vet Med Assoc 1982, 180:519-522.

4. Hansen HJ: A pathologic-anatomical study on disc degeneration in dogs. Acta Orthop Scand Suppl 1952:I-I I7.

5. Havranek-Balzaretti B: Beitrag zur Aetiologie der Dackellähme und Vorschlag zur züchterischen Selektion. (The etiology of intervertebral disc disease in the dachshund and proposal of an eradication programme). Zürich: Veterinär-Chirurgischen Klinik und Institut für Veterinärpatologie. Dissertation, Universität Zürich; 1980.

6. Stigen $\varnothing$ : Calcification of intervertebral discs in the dachshund. A radiographic study of II5 dogs at I and 5 years of age. Acta Vet Scand 1996, 37:229-237.

7. Stigen $\varnothing$ : Calcification of intervertebral discs in the dachshund. A radiographic study 327 young dogs. Acta Vet Scand 1991, 32:197-203.

8. Stigen $\varnothing$, Christensen $\mathrm{K}$ : Calcification of intervertebral discs in the dachshund. An estimation of heritability. Acta Vet Scand 1993, 34:357-361.

9. Jensen VF, Christensen KA: Inheritance of disc calcification in the dachshund. J Vet Med A Physiol Pathol Clin Med 2000, 47:331-340.

10. Seiler G, Häni H, Scheidegger J, Busato A, Lang J: Staging of lumbar intervertebral disc degeneration in nonchondrodystrophic 
dogs using low-field magnetic resonance imaging. Vet Radiol Ultrasound 2003, 44: 179-184.

II. Bray JP, Burbidge HM: The canine intervertebral disk. Part two: degenerative changes - nonchondrodystrophoid versus chondrodystrophoid disks. J Am Anim Hosp Assoc 1998, 34:135-144.

12. Jensen VF, Arnbjerg J: Development of intervertebral disk calcification in the dachshund: a prospective longitudinal radiographic study. J Am Anim Hosp Assoc 200I, 37:274-282.

13. Morgan JP, Silverman S: Techniques of veterinary radiography 3rd edition. Davis, Veterinary Radiology Associate; 1982:190-195.

14. Burk RL, Ackerman N: Small animal radiology. A diagnostic atlas and text New York, Churchill Livingstone; 1986:34I-373.

15. Culling CFA, Allison RT, Barr WT: Cellular pathology technique 4th edition. London, Butterworth \& Co; |985:4|7-4| 9.

16. Kirberger RM: Spine - general. In BSAVA manual of canine and feline musculoskeletal imaging Edited by: Barr FJ, Kirberger RM. Quedgeley, BSAVA; 2006:220-232

17. Morgan JP, Ljunggren G, Read R: Spondylosis deformans (vertebral osteophytosis) in the dog. I Small Anim Pract 1967, 8:57-66.

18. Done SH, Drew RA, Robins GM, Lane JG: Hemivertebra in the dog: clinical and pathological observations. Vet Rec 1975, 96:313-317.

Publish with Bio Med Central and every scientist can read your work free of charge

"BioMed Central will be the most significant development for disseminating the results of biomedical research in our lifetime. "

Sir Paul Nurse, Cancer Research UK

Your research papers will be:

- available free of charge to the entire biomedical community

- peer reviewed and published immediately upon acceptance

- cited in PubMed and archived on PubMed Central

- yours - you keep the copyright

Submit your manuscript here:

http://www.biomedcentral.com/info/publishing_adv.asp
BiolMedcentral 